Current Definitions of Central Line-Associated Bloodstream Infection: Is the Emperor Wearing Clothes? •

Author(s): Daniel J. Sexton, MD, Luke F. Chen, MBBS, MPH, CIC, FRACP, Deverick J. Anderson, MD, MPH

Source: Infection Control and Hospital Epidemiology, Vol. 31, No. 12 (December 2010), pp. 1286-1289

Published by: The University of Chicago Press on behalf of The Society for Healthcare Epidemiology of America

Stable URL: http://www.jstor.org/stable/10.1086/657583

Accessed: 10/06/2011 08:02

Your use of the JSTOR archive indicates your acceptance of JSTOR's Terms and Conditions of Use, available at http://www.jstor.org/page/info/about/policies/terms.jsp. JSTOR's Terms and Conditions of Use provides, in part, that unless you have obtained prior permission, you may not download an entire issue of a journal or multiple copies of articles, and you may use content in the JSTOR archive only for your personal, non-commercial use.

Please contact the publisher regarding any further use of this work. Publisher contact information may be obtained at http://www.jstor.org/action/showPublisher?publisherCode=ucpress.

Each copy of any part of a JSTOR transmission must contain the same copyright notice that appears on the screen or printed page of such transmission.

JSTOR is a not-for-profit service that helps scholars, researchers, and students discover, use, and build upon a wide range of content in a trusted digital archive. We use information technology and tools to increase productivity and facilitate new forms of scholarship. For more information about JSTOR, please contact support@jstor.org. 


\title{
Current Definitions of Central Line-Associated Bloodstream Infection: Is the Emperor Wearing Clothes?
}

\author{
Daniel J. Sexton, MD; Luke F. Chen, MBBS, MPH, CIC, FRACP; Deverick J. Anderson, MD, MPH
}

As has happened at many other institutions, our 750-bed tertiary care hospital has seen rates of central line-associated bloodstream infection (CLABSI) decrease dramatically in intensive care units (ICUs). Overall rates of CLABSI dropped 10 -fold, from 4.4 to 0.44 cases per 1000 central-line days, in our 6 adult ICUs in the past 24 months. Five years ago, most cases of CLABSI in our ICUs were due to gram-positive cocci such as Staphylococcus aureus. During the past 12 months, only 1 case of CLABSI due to $S$. aureus occurred in our adult ICUs, each of which cares for 16-20 patients per day.

There are several potential reasons for this remarkable decrease in the incidence of CLABSI. Medical and nursing leaders in our ICUs mandated the use of a central-line "insertion bundle." Computer-based training and certification on central-line insertion and maintenance are required for all healthcare staff in ICUs. Leadership teams in each unit have enforced hand hygiene compliance through a rigorous compliance-monitoring program. ${ }^{2}$ Time-trend analyses of unit-specific rates of device-related infections are part of our hospital's "dashboard" of quality indicators. Furthermore, each unit now posts a sign prominently displaying the number of days since its last case of CLABSI.

Team culture and leadership attitudes have also changed in our ICUs. Medical and nursing leadership teams now receive real-time (ie, immediate) notification of any devicerelated healthcare-associated infection (HAI). They review the circumstances of each HAI that occurs in their unit. These leaders firmly believe that they are directly responsible for prevention of HAIs - an attitude encouraged and fostered by our hospital's administrative leadership and our infection prevention team.

The preceding changes in education, process measures, culture, and leadership attitudes have improved outcomes for patients. However, as with other things in life, new problems are often the price of progress. The new problem that we will focus on in this discussion is an inadequate definition of CLABSI.

It appears that the surveillance definition for CLABSI from the Centers for Disease Control and Prevention (CDC) originally aimed to be highly sensitive to capture all cases of CLABSI. Unfortunately, its specificity was a secondary concern. In the past, when rates of CLABSI were higher in most American hospitals, hospital epidemiologists would review the CLABSI rate with the understanding that there was unavoidable "data noise" because of the highly sensitive and poorly specific definition. This lack of specificity in the definition of CLABSI is an old problem that has moved to the forefront of our awareness for 2 important reasons. First, external pressures to improve safety outcomes are a concern for all hospitals. This pressure comes from national directives, mandated public reporting, and "pay for performance" that is tied to specific process measures. Second, now that the incidence of CLABSI has substantially decreased, hospitals are realizing that "getting to zero" (ie, reducing the incidence to zero) is implausible, if not impossible, with the current definition of CLABSI. ${ }^{3}$ Additionally, a series of secondary problems and consequences emerge from flaws of the existing definition. These problems and consequences will continue or worsen unless the definition is modified.

The consequences of an inadequate definition of CLABSI are illustrated by a dispute that arose at our institution when a physician from the surgical ICU protested that a patient we identified as having CLABSI did not have this condition. He argued that the erroneous classification of this HAI as a case of CLABSI would damage morale in his unit and undermine confidence in other surveillance data we collect and disseminate. The details of this case are as follows.

A woman was admitted to an ICU with hypotension and intraabdominal injuries following a car accident. A central line was inserted to provide fluid resuscitation and blood product replacement. A laparotomy revealed liver lacerations that required hepatorrhaphy, packing, and angiographic embolization of gastroduodenal vessels. There were no visible injuries to her bowel. She subsequently underwent 2 additional laparotomies. In the first procedure, a gangrenous gallbladder and suspected bowel ischemia led to a cholecystectomy and segmental imbrication of the

From the Duke University Medical Center, Durham, North Carolina (D.J.S.)

Received May 12, 2010; accepted June 16, 2010; electronically published October 27, 2010.

Infect Control Hosp Epidemiol 2010; 31(12):1286-1289

(C) 2010 by The Society for Healthcare Epidemiology of America. All rights reserved. 0899-823X/2010/3112-0012\$15.00. DOI: 10.1086/657583 
transverse colon. During a second laparotomy, she underwent repair of the left hemi-diaphragm and a small perforation of the stomach (thought to be due to migration of a pigtail catheter). The surgeons found no macroscopic evidence of established infection and thus, appropriately, did not collect specimens for microbiologic testing. Cultures of blood drawn from peripheral veins 1 day after surgical repair grew Pantoea agglomerans and Proteus mirabilis.

Our infection preventionists applied the National Healthcare Safety Network (NHSN) definition for CLABSI ${ }^{4}$ and were unable to label this patient's bloodstream infection (BSI) as secondary to another source, as there was no clear evidence of infection and no pus was encountered at any of the patient's surgeries. Furthermore, there were no pathogens cultured from specimens collected from any site to indicate that an infection arose from the gastrointestinal tract or abdomen, even though there were compelling clinical reasons to suggest that this had occurred. Our infection preventionists argued that failure to follow the "CDC definitions" would make our hospital's data "dishonest." Moreover, failure to include this infection as a case of CLABSI would undermine their authority and hurt the morale of our infection control team.

We believe that this case and many other cases of gramnegative bacteremia or fungemia in seriously ill patients in ICUs likely occur either because of translocation of microorganism from edematous, abnormal, or adynamic segments of bowel or because of microscopic or macroscopic defects in the bowel wall. As in the case we describe, absence of proof (of an established infection at a site other than a central vascular catheter as a source of bacteremia or fungemia) cannot be cited as proof of absence. In fact, the origin of some episodes of bacteremia or fungemia is impossible to determine. To assign, by default, all such BSIs to a category of "central-line associated" simply because a central line has been inserted is not only folly, it is also intellectually and operationally incorrect.

There is compelling evidence that spontaneous gut-associated bacteremia and fungemia occur in seriously ill or otherwise immunocompromised patients. Gut-associated BSIs can also arise from gastrointestinal procedures that have or do not have associated localized suppuration. Specifically, bacterial translocation is known to occur after abdominal surgery $^{5-8}$ (at higher rates if there is colon manipulation) ${ }^{9}$ in patients with underlying bowel disease ${ }^{10}$ and in critically ill patients, even if there is no bowel manipulation or preexisting bowel disease. ${ }^{11,12}$

Hospital epidemiologists and infection preventionists now face an unnecessary dilemma when adjudicating whether a patient with bacteremia has a CLABSI or a secondary BSI. As a result of the issues raised above, we suspect that criteria used to determine whether there is a secondary source of infection vary from hospital to hospital. Indeed, it is likely that some epidemiologists would conclude on the basis of circumstantial evidence that our patient who had Pantoea and Proteus organisms recovered from blood had bacteremia secondary to a peritoneal infection. However, other hospital epidemiologists might strictly apply existing criteria and come to the opposite conclusion. In fact, informal discussions with other hospital epidemiologists have led us to believe that such adjudication based on circumstantial evidence and intuition is commonly but nonuniformly applied at individual hospitals. Such unnecessary subjectivity undermines the utility and reliability of publicly reported data on rates of CLABSI.

The current NHSN definition of CLABSI is also deficient because some microorganisms that are likely to be contaminants are considered to be pathogens. Specifically, we believe the inclusion in the definition of laboratory-confirmed BSI of the criterion that a single blood culture positive for enterococci can be considered recovery of a "recognized pathogen" rather than as a "common skin contaminant" is incorrect. This issue is well illustrated by the following case from our institution.

A woman with diabetes mellitus was admitted with Streptococcus pneumoniae meningitis and sepsis. She required ICU admission, intubation, and, over the course of her illness, insertion of 2 central lines. A percutaneous endoscopic gastrostomy tube was placed for enteral feeding. She had intermittent fever over the ensuing 7 days, and the initial central lines were replaced. During this time, results of several blood and urine cultures were negative for pathogens. Imaging studies and serial clinical examinations revealed no localizing features of an infection. Ultimately, 1 of 2 cultures of blood samples collected on day 15 of hospitalization grew coagulase-negative Staphylococcus species and Enterococcus faecalis. Antibiotic therapy active against both organisms was started. An infectious diseases specialist who evaluated this patient concluded, however, that the single positive blood culture result represented a contaminant and recommended discontinuation of antimicrobial therapy. Results were negative for 4 subsequent cultures of blood samples obtained while the patient was receiving antibiotic therapy, and results were also negative for 6 additional blood cultures performed after antimicrobial therapy was discontinued.

Once again, our infection preventionists followed the NHSN definition of CLABSI in designating this patient's illness as a case of CLABSI. They noted that E. faecalis was recovered from the blood of a patient with a central line and pointed out that no alternative site of infection could be determined. The leadership of the ICU team caring for this patient was incredulous about this assignment. They cited the consulting infectious diseases expert's assessment as evidence that the single positive blood culture result represented contamination and not infection. We ultimately agreed with the ICU team and the conclusions of the infectious disease specialist who saw this patient. Our infection preventionists argued that failure to adhere to "the CDC's definitions" would undermine our unit's credibility and integrity. Again, the morale of the infection control team was damaged by our decision to side with the ICU team. 
The evidence that enterococci are frequent contaminants of blood cultures is compelling. ${ }^{13-15}$ Indeed, infectious diseases specialists routinely perform a repeat blood culture as a first step to assess patients who have a single blood culture positive for enterococci. If the repeat culture result is negative, most infectious diseases specialists would conclude that the single positive blood culture result represents contamination. ${ }^{16,17}$

The issue of using a single blood culture positive for enterococci as evidence of a laboratory-confirmed case of CLABSI is not a trivial one. We retrospectively reviewed 1,187 episodes of enterococcal "bacteremia" that occurred during a 4-year period using data from 11 hospitals in the Duke Infection Control Outreach Network. In total, 802 (68\%) of 1,187 patients had only 1 blood culture positive for enterococci. ${ }^{18}$ Furthermore, 469 (58\%) of these single-positive-culture CLABSI events were polymicrobial; most commonly, other skin contaminants were isolated, such as coagulasenegative staphylococci. Of the 385 patients with more than 1 positive blood culture result during the index hospitalization, only 307 patients $(26 \%$ of the whole cohort of 1,187 ) had 2 or more blood cultures positive for enterococci within a contiguous 48 -hour period.

The time for a change is now, because the CLABSI rate has emerged as an important performance measure. This rate is widely used to perform time-trend analysis on performance within individual units and to compare performance between units within a hospital and between hospitals. Erroneous data that overestimate rates of CLABSI can damage morale and lead to false conclusions about quality of care. Erroneously attributing a BSI to a vascular device damages the credibility of our infection prevention team and undermines the credibility of all the data we collect and disseminate. Finally, incorrect data on rates of CLABSI decrease, if not eliminate, the utility of publicly reported data on the incidence of these infections.

In view of the fact that the source of some BSIs cannot be accurately determined, what alternatives to the current definitions are appropriate and practical? We believe several alternatives exist and propose 2 changes to the current surveillance definition for CLABSI: (1) inclusion of an "indeterminate source" label for some BSIs, and (2) modification of the current NHSN definition of CLABSI to acknowledge that a single blood culture positive for enterococci has little clinical significance, similar to the interpretation of a single blood culture positive for other "common skin contaminants," such as coagulase-negative staphylococci.

Thus, we believe that CLABSIs can reasonably be divided into 3 categories: primary, secondary, or indeterminate source. Criteria for an indeterminate category could be designed to cover situations similar to the first case described above. Such a designation should include clinical criteria pointing to a possible source of a BSI, such as bowel surgery, perforation, or other organ blockage or instrumentation, even if a positive culture result for a specimen from such a site is absent. Hospitals could and should continue to monitor their rates of primary, secondary, and indeterminate-source CLABSIs as quality measures and as a way to document outcomes after prevention efforts.

Acceptance of an "indeterminate source" category for some patients with BSI would prevent many of the unintended consequences described above. Furthermore, this label would allow hospital epidemiologists to admit something that every good clinician already knows: we can not determine the source of every infection with certainty. Finally, local staff, administrative leaders, and patients would be less likely to assume that infections of indeterminate source were the result of inadequate or improper care.

We have already modified our surveillance definition for CLABSI to include enterococci as a common skin contaminant. Other surveillance definitions developed by the CDC and NHSN have been "officially" modified in the past. For example, 2 key changes were made to the definition of laboratory-confirmed BSI in the past decade. First, infections with onset in the community were subdivided into "healthcare-associated infection" and "community-acquired infection." ${ }^{19}$ Second, the NHSN modified the definition of CLABSI in 2008 by excluding patients who had a single blood culture positive for coagulase-negative staphylococci, regardless of whether the patient received antibiotic treatment active against this organism. Furthermore, a precedent for including an "indeterminate source" of infection was set when the NHSN modified the surveillance definitions in the Clostridium difficile infection module. ${ }^{20}$ The label "indeterminate source" does not imply that inadequate investigation was performed, but instead concedes that our knowledge of infections has limitations.

To our knowledge, the sensitivity and specificity of the current NHSN definition of CLABSI have not been rigorously assessed. In this respect, the CDC is somewhat like the Emperor in Hans Christian Anderson's fable "The Emperor's New Clothes." The public all pretended that the Emperor's clothes were beautiful until a child declared "[the Emperor] isn't wearing anything!" Similarly, the current NHSN definition for CLABSI cannot be assumed to be accurate because the CDC simply declares them to be accurate and because the public and mainstream medical communities accept them as accurate. There is too much at stake to rely on nonvalidated definitions when rates of these infections affect a hospital's finances and reputation. It is not honest nor realistic to assume that all CLABSIs will be eliminated or that we will "get to zero" using the current definition for CLABSI. In fact, this phrase seems eerily similar to and likely as implausible as the statement often attributed to Surgeon General William Stewart that we can "close the book on infectious diseases." ${ }^{21}$

In summary, we believe improvements to the definition of CLABSI can, should, and, in fact, must be made. Allowing some BSIs to be categorized as of indeterminate source, and including enterococci as a common skin contaminant, will allow epidemiologists to maintain their credibility with clinicians. Furthermore, surveillance data concerning infections 
categorized as of indeterminate source will be more epidemiologically and clinically useful than data derived from current definitions, which are inconsistent with common clinical practice. Finally, we believe it is important that research focused on the validation of all existing surveillance definitions be undertaken. If the CDC and NHSN are not inclined to lead this effort, then the Society for Healthcare Epidemiology of America should promote such initiatives. The findings for such research will help us determine whether the Emperor's clothes are real or imagined and it will help others to accept the data we collect as useful and accurate.

\section{ACKNOWLEDGMENTS}

Potential conflicts of interest. L.F.C. reports he has received research grant funding from Merck and travel grants from AstraZeneca and GlaxoSmithKline. D.J.A. reports he has received research grant funding from Pfizer, Merck, and the Robert Wood Johnson Foundation. D.J.S. reports no conflicts of interest relevant to this article.

Address reprint requests to Daniel J. Sexton, MD, FACP, Box 102359, Duke University Medical Center, Durham, North Carolina, 27710 (sexto002 @mc.duke.edu).

\section{R E F E R E N C E S}

$\rightarrow$ 1. Pronovost P, Needham D, Berenholtz S, et al. An intervention to decrease catheter-related BSIs in the ICU. N Engl J Med 2006;355(26):2725-2732.

2. Cooper LM, Oden MA, Elliott BK, Harger JA, Anderson DJ. Increased hand hygiene observations add validity to infection control data-but how to achieve in a busy hospital world? In: Program and abstracts of the Fifth Decennial International Conference on Healthcare-Associated Infections; Atlanta, GA; 2010.

$\rightarrow$ 3. Shuman EK, Washer LL, Arndt JL, et al. Analysis of central line-associated BSIs in the intensive care unit after implementation of central line bundles. Infect Control Hosp Epidemiol 2010;31(5):551-553.

4. National Healthcare Safety Network (NHSN). Central line-associated BSI (CLABSI) event. Updated June 2010. http://www.cdc.gov/nhsn/TOC _PSCManual.html. Accessed October 13, 2010.

5. O’Boyle CJ, MacFie J, Mitchell CJ, Johnstone D, Sagar PM, Sedman PC. Microbiology of bacterial translocation in humans. Gut 1998;42(1):2935 .
6. MacFie J, O’Boyle C, Mitchell CJ, Buckley PM, Johnstone D, Sudworth P. Gut origin of sepsis: a prospective study investigating associations between bacterial translocation, gastric microflora, and septic morbidity. Gut 1999;45(2):223-228.

7. Reddy BS, MacFie J, Gatt M, Macfarlane-Smith L, Bitzopoulou K, Snelling AM. Commensal bacteria do translocate across the intestinal barrier in surgical patients. Clin Nutr 2007;26(2):208-215.

$\rightarrow$ 8. Reddy BS, Gatt M, Sowdi R, MacFie J. Surgical manipulation of the large intestine increases bacterial translocation in patients undergoing elective colorectal surgery. Colorectal Dis 2006;8(7):596-600.

$\rightarrow$ 9. Woodcock NP, Sudheer V, El-Barghouti N, Perry EP, MacFie J. Bacterial translocation in patients undergoing abdominal aortic aneurysm repair. Br J Surg 2000;87(4):439-442.

10. Turner JR. Intestinal mucosal barrier function in health and disease. Nat Rev Immunol 2009;9(11):799-809.

11. Stechmiller JK, Treloar D, Allen N. Gut dysfunction in critically ill patients: a review of the literature. Am J Crit Care 1997;6(3):204-209.

12. Van Leeuwen PA, Boermeester MA, Houdijk AP, et al. Clinical significance of translocation. Gut 1994;35(1 Suppl):S28-S34.

13. Weinstein MP, Towns ML, Quartey SM, et al. The clinical significance of positive blood cultures in the 1990s: a prospective comprehensive evaluation of the microbiology, epidemiology, and outcome of bacteremia and fungemia in adults. Clin Infect Dis 1997;24(4):584-602.

14. Weinstein MP, Reller LB, Murphy JR, Lichtenstein KA. The clinical significance of positive blood cultures: a comprehensive analysis of 500 episodes of bacteremia and fungemia in adults. I. Laboratory and epidemiologic observations. Rev Infect Dis 1983;5(1):35-53.

$\rightarrow$ 15. Beezhold DW, Slaughter S, Hayden MK, et al. Skin colonization with vancomycin-resistant enterococci among hospitalized patients with bacteremia. Clin Infect Dis 1997;24(4):704-706.

16. Hall KK, Lyman JA. Updated review of blood culture contamination. Clin Microbiol Rev 2006;19(4):788-802.

17. Weinstein MP. Blood culture contamination: persisting problems and partial progress. J Clin Microbiol 2003;41(6):2275-2278.

18. Chen LF, Freeman JT, Sexton DJ, Choi YI, Anderson DJ. NHSN definition of laboratory-detected BSI is overly sensitive for Enterococcus. In: Program and abstracts of the 19th Annual Scientific Meeting of the Society for Healthcare Epidemiology of America (SHEA); San Diego, CA; 2009.

19. Friedman ND, Kaye KS, Stout JE, et al. Health care-associated BSIs in adults: a reason to change the accepted definition of community-acquired infections. Ann Intern Med 2002;137(10):791-797.

20. McDonald LC, Coignard B, Dubberke E, Song X, Horan T, Kutty PK. Recommendations for surveillance of Clostridium difficile-associated disease. Infect Control Hosp Epidemiol 2007;28(2):140-145.

21. Sassetti CM, Rubin EJ. The open book of infectious diseases. Nat Med 2007;13(3):279-280. 